\title{
El escepticismo de la comunicación incómoda. Las herramientas claves en la búsqueda del éxito en la comunicación medioambiental: un análisis de las piezas de comunicación de Al Gore y Bjorn Lomborg
}

\author{
Vinicius VALENTE BAYMA \\ Asistente de Proyectos en EUREC Agency \\ viniciusbayma@gmail.com
}

Recibido: $23 / 04 / 2012$

Aceptado: 30/10/2012

\begin{abstract}
Resumen
Las Naciones Unidas ha formado parámetros que evidencian que el planeta se encuentra en una crisis ambiental sin precedentes en la historia de la humanidad. Sin embargo la imprecisión y diversidad de los datos publicados sobre el cambio climático generan preocupación y desinterés por parte de los políticos y del público general. Este trabajo tiene como objetivo contribuir a la mejora de la comunicación medioambiental y facilitar la difusión correcta por los medios de las características de los problemas relacionados al tema y su comprensión. Por lo tanto, investiga las particularidades presentes en los libros/documentales Una verdad incómoda de Al Gore y Cool it de Bjorn Lomborg. También evalúa el libro The Skeptical Environmentalist escrito por Lomborg y analiza los argumentos y la utilización de herramientas comunicacionales relevantes.
\end{abstract}

Palabras clave: Comunicación, Medioambiental, Gore, Lomborg, Medios

\section{The Skepticism of the Inconvenient Communication. The Key Issues Leading to Success in the Environmental Communication: An Analysis of Al Gore's and Bjorn Lomborg's Pieces}

\begin{abstract}
The United Nations has been providing reliable data that shows that the planet lies on an environmental crisis never seen before in the history of mankind. However, the inaccuracy and diversity of the published data on climate change make policy makers and the general public anxious and indifferent. This paper intends to contribute with the improvement of the environmental communications and ease correct diffusion in the media of the issues related to the topic and its understanding by decision makers. In order to do so, this study investigates the particularities of the book/documentary An inconvenient truth by $\mathrm{Al}$ Gore and also of the book The Skeptical Environmentalist, by Bjorn Lomborg, together with his book/documentary Cool it. The paper analyses the arguments and the use of relevant communications tools.
\end{abstract}

Keywords: Communication, Environmental, Gore, Lomborg, Media

\section{Referencia normalizada}

VALENTE BAYMA, Vinicius (2013): "El escepticismo de la comunicación incómoda. Las herramientas claves en la búsqueda del éxito en la comunicación medioambiental: un análisis de las piezas de comunicación de Al Gore y Bjorn Lomborg”. Estudios sobre el mensaje periodístico. Vol. 19, núm. 1, págs.: 311-328. Madrid, Servicio de Publicaciones de la Universidad Complutense.

Sumario: 1. Introducción. 2. La verdad incómoda de Al Gore; 2.1 Biografía; 2.2 La verdad incómoda; 2.3 Críticas. 3. Bjorn Lomborg y la batalla contra el Apocalipsis; 3.1 Biografía; 3.2 La investigación del estado "real" del mundo; 3.3 El mensaje tranquilizante de Cool it; 3.4 Críticas. 4. Consideraciones finales. 5. Referencias bibliográficas. 


\section{Introducción}

El concepto de comunicación científica masiva está relacionado con la emisión de informaciones sobre ciencia para un público general mayoritariamente formado por personas no científicas. Este proceso envuelve muchas veces a científicos formados, pero ha evolucionado para un campo profesional propio que incluye, entre otras cosas, políticas científicas, producción mediática, periodismo y divulgación científica.

A partir de la segunda mitad del siglo XX, una subárea particular de la comunicación científica pasó a atraer fuertemente la atención de la sociedad internacional debido a un fuerte cambio y la mejora de la comunicación sobre el tema. La Organización de las Naciones Unidas ya tiene formados parámetros que evidencian que el planeta se encuentra en una crisis ambiental sin precedentes en la historia de la humanidad. Crisis que se ha vuelto relevante no solamente por resultar en posibles cambios climáticos alrededor del globo, sino que también en virtud de la posibilidad de alterar drásticamente la calidad de vida de los seres humanos, sobre todo los más pobres.

Sobre este problema, existen, actualmente, dos puntos de vista mayoritarios y directamente opuestos. El primero es apocalíptico y defiende que el futuro de la civilización humana está en peligro debido al modelo insostenible de desarrollo adoptado por la sociedad. Esta versión es compartida por diversos científicos y organizaciones en todo el planeta y alcanzó gran visibilidad debido al político estadounidense Al Gore y su libro/documental Una verdad incómoda. La otra visión es liderada por Bjorn Lomborg, conocido como el ambientalista escéptico por el título de su libro. El danés usa la estadística para mostrar, entre otros temas, que la situación actual del clima no está tan mal como dicen los climatólogos y que, en realidad, el escenario está mejorando. Lomborg defiende lo que sería el "estado real del mundo" y que las decisiones con respecto al medio ambiente deberían ser tomada llevando estos datos en cuenta.

El tema de estudio de este artículo es la investigación sobre las particularidades presentes en las obras utilizadas por Al Gore y Bjorn Lomborg y los argumentos utilizados por ambos para divulgar sus ideas.

Se establece como objetos principales de estudio el libro/documental Una verdad incómoda de Al Gore, ganador del Premio Nobel de la Paz en 2007 y el libro The Skeptical Environmentalist, de Bjorn Lomborg, junto con su libro/documental Cool it, que combate las ideas sobre el medio ambiente difundidas por Al Gore.

Con la investigación se pretende analizar la manera con la que los dos personajes utilizaron las herramientas de la comunicación para difundir su mensaje para contestar a la siguiente problemática: ¿Cuál es la mejor manera de transmitir el mensaje ambiental en los medios teniendo como objetivo la concientización de la sociedad para mitigar los efectos del cambio climático?

El objetivo general de la investigación es identificar la base de los argumentos, las herramientas y el discurso utilizado por Gore y Lomborg para identificar la razón de su enorme éxito comunicacional en el área de la comunicación medioambiental y analizar sus respectivas piezas utilizadas para difundir sus ideas. Se espera que al final, sea posible identificar algunas herramientas claves para comunicar de la mejor manera temas relacionados al medio ambiente y el cambio climático. 
Metodológicamente la presente investigación se clasifica cuanto a los medios de naturaleza bibliográfica, en la medida en que se busca en libros y artículos la fundamentación teórica que le sirve de base y como documental, mientras utiliza las piezas identificadas como principales como objetos de análisis. Las obras de Al Gore serán analizadas en la primera parte y luego cederán la vez al análisis de las piezas de Bjorn Lomborg.

Se justifica el tema de la investigación por la oportunidad de contribuir con la mejora de la comunicación medioambiental. La difusión rápida y sencilla de los conocimientos asociados al medio ambiente y al cambio climático son urgentes debido a la necesidad de se establecer un nuevo acuerdo internacional de reducción de gases de efecto invernadero para mitigar rápidamente las consecuencias del calentamiento global.

Esperase que la lectura de este trabajo proporcione momentos igualmente agradables cuanto los que el proceso de elaboración representaron para este investigador.

\section{La verdad incómoda de Al Gore}

"El futuro de la civilización humana está en peligro"

Al Gore

\subsection{Biografía}

El Vicepresidente de los Estados Unidos durante la administración de Bill Clinton (1993-2001) Albert Arnold Gore Jr es hoy director de la Current TV, un canal a cable y satélite independiente hecho para jóvenes y basado en contenidos creados por el público y periodismo ciudadano. Nacido en Washington en 1948, actúa también como director de la Generation Investment Management, una compañía que se ocupa de un nuevo enfoque para las inversiones sostenibles. Además, es Profesor Visitante en la Universidad Middle Tennessee State y comanda la "Alliance for Climate Protection", una organización sin animo de lucro organizada para ayudar a solucionar los problemas relacionados a la crisis climática (The Nobel Foundation, 2007: web).

$\mathrm{Su}$ trayectoria política es bastante conocida mundialmente. Miembro del partido demócrata estadounidense, fue elegido para la Cámara de Diputados de Estados Unidos en 1976, 1978, 1980 y 1982 y para el Senado en 1984 y 1990. Tres años más tarde asumió la vicepresidencia del país durante los ocho años del gobierno de Clinton, periodo en que también fue miembro central del panel económico del presidente y lideró las acciones del Gobierno relacionadas a la protección del medio ambiente. Además, actuó como Presidente del Senado y miembro del Consejo de Seguridad Nacional (Take Part, 2007: web).

Como autor, firma los "best sellers" Earth in the Balance (1992) y Una verdad incómoda (2006), con lo cual ganó el Premio Nobel de la Paz en 2007, junto al IPCC por informar el mundo sobre los peligros impuestos por el cambio climático.

En 2000 fue candidato a la presidencia estadounidense por el partido demócrata y tuvo como adversario el republicano George W. Bush. En una elección marcada por una cuenta polémica de votos, Gore obtuvo más votos populares pero Bush salió vencedor debido a su numero superior de delegados en el colegio electoral. 
Tras las elecciones de 2000, Gore decidió volver a dar la presentación sobre calentamiento global que solía realizar en los años sus años de vicepresidente.

En los primeros seis años del siglo XXI, Al Gore viajó por todo el mundo, intercambiando información con "cualquier persona que quisiera escuchar" (Take Part, 2007: web) en universidades, pueblos y ciudades. En 2005, realizó una presentación en Los Angeles para un grupo reunido por la activista Laurie David. Luego, ella y el productor Lawrence Bender sugirieron a Gore que hiciera una película basada en su presentación. En principio, la idea le pareció un poco absurda y el político no veía como sus dispositivas podrían verse en película. Pero enseguida los productores fueron a otra presentación con Jeff Skoll, director de la productora Participant Productions, que mostró interés en el proyecto. A partir de ahí, Gore fue presentado al cineasta Davis Guggenheim, que estaba interesado en dirigir el proyecto, Scott Burns y Lesley Chicott, que asumieron el equipo de producción.

\subsection{La verdad incómoda}

La principal preocupación de Al Gore con el proyecto era que la adaptación de la presentación en una película no sacrificase la ciencia por el entretenimiento. Pero de poco en poco, el político se fue convenciendo y confiando en el equipo que parecía estar comprometido con los mismos objetivos. Luego, Gore se dio cuenta que para atingir rápidamente un gran número de personas, y no sólo algunas decenas por noche, una película, que luego se llamaría Una verdad incómoda, sería la mejor manera para hacerlo (Gore, 2006a: 4).

Dirigido por Davis Guggenheim, el documental Una verdad incómoda muestra la ideología verde de Al Gore en su jornada que tiene el reto de detener el progreso del cambio climático a través de la exposición de sus mitos y conceptos erróneos que rondan el término. La película es un retrato íntimo del político y su palestra itinerante sobre cambio climático que mezcla alertas y bromas con información e intenta comunicar el problema de forma sencilla, graciosa, abierta pero también preocupada y alarmada con la "emergencia planetaria".

El documental muestra las previsiones apocalípticas de un supuesto futuro mas cálido a través de la jornada personal de Gore, desde un idealista universitario que vio el nacimiento de una crisis ambiental, se convirtió en un joven Senador que enfrenta una tragedia familiar que alteró su perspectiva hasta el hombre que casi llegó a ser Presidente y decidió retornar a la "causa más importante de su vida". La película intenta mostrar que el cambio climático ya no es mas un problema político sino que también un desafío moral grande que la civilización debe enfrentar.

Se trata de una película bastante didáctica, que muestra a través de gráficos, fotos y estudios el problema de el calentamiento global. Tiene la clara intención de alertar el público sobre los problemas por los cuales el planeta pasa.

Tras su estreno en el Sundance Film Festival en 2006 y una exhibición internacional en Cannes, Una verdad incómoda se volvió conocido mundialmente. En el mismo año ganó el Oscar de mejor documental y mejor música. Además, se convirtió en un fenómeno mundial y en uno de los documentales más vistos en el mundo, con una audiencia mundial estimada en 5 millones de personas. 
El impacto alcanzado por Una verdad incómoda fue bastante amplio. Desde su estreno en 2005, la película ayudó a llamar la atención de gobernantes, líderes, organizaciones y individuos por todo el mundo y a tomar actitudes con respecto al calentamiento global. Gracias al documental, cerca de 106.000 toneladas de carbono fueron compensadas en el año siguiente al estreno y otras 4.200 toneladas fueron compensadas sólo por las personas que pasaron a utilizar bombillas fluorescentes. Además, el tema cambio climático alcanzó más de un mil millones de personas y cerca de 2600 personas fueron entrenadas para hacer la presentación de la organización Climate Progress, que, por consecuencia, ayudo en la concientización de 4 millones de personas. En los Estados Unidos, el Presidente Barack Obama creó una nueva posición de asistente del gobierno para el Clima y Energía, mientras la Cámara de Diputados y el Senado establecieron un comité para independencia energética y calentamiento global. Inglaterra, Escocia, la Republica Checa, Nueva Zelandia, Alemania y la Columbia Británica en Canadá incorporaron el documental en el programa de sus escuelas (Take Part, 2007: web).

En la película, $\mathrm{Al}$ Gore afirma que la crisis climática es verdaderamente peligrosa y que ahora las evidencias muestran que si la humanidad no actúa de manera fuerte y rápida, el planeta enfrentará catástrofes terribles, como el huracán Katrina. Según el autor, la capa de hielo del polo norte esta derritiendo así como diversos glaciares montañosos en el mundo. La masiva cantidad de hielo en Groenlandia se esta desestabilizando junto con el hielo presente en la cima de las islas de la parte oeste de la Antártica. La lista de fenómenos que están en peligro debido al calentamiento global también incluye la configuración de los océanos y de corrientes de viento que mantienen las mismas características desde hace 10 mil años. Sin embargo, el documental, que tiene un título bastante cautivante y llamativo, no sólo hace alertas, sino que también sugiere lo que se puede hacer para mitigar las consecuencias del cambio de la temperatura global e intenta señalar las oportunidades que una crisis como esta puede ofrecer. Esto seguramente fue un punto positivo para Al Gore y es una de las causas que llevaron al gran éxito sus piezas de comunicación, pues impide el desarrollo de un sentimiento de incapacidad o conformismo en los espectadores. En general, tras escuchar la expresión "el poder de cambio esta en las manos de cada uno", el público se siente competente, parte de un sistema y unido por una causa mayor. El documental afirma que la actual crisis incluye nuevos trabajos y formas de hacer negocios como el desarrollo de las energías renovables y la creación de medidas de eficiencia energética. Para Gore, la crisis actual también ofrece la oportunidad de vivir experiencias que muy pocas generaciones en la historia tuvieron el privilegio de vivenciar: una misión generacional, la satisfacción de actuar con un propósito moral, una causa compartida que genera unión global, la emoción de ser forzado por las circunstancias a dejar de lado la pequeñez y el conflicto que muchas veces ahoga la incansable necesidad humana de transcenderse, la oportunidad de ascender.

Se puede afirmar que el protagonista de Una verdad incómoda contribuyó bastante al éxito del documental. El personaje visto no es un político intocable en su torre de marfil. Sea por su practica de años en el mundo de discursos y debates políticos, o por la experiencia de haber hecho la misma presentación varias veces, lo que se ve en la pe- 
lícula es un personaje seguro, tranquilo, cortés y educado. El espectador ve Gore como una persona cercana, común y comprometida con su causa. Se nota que es alguien que se siente cómodo al hablar a diversas personas y también con el hecho de estar siendo filmado. El político es fotogénico, tiene buena apariencia y sabe moverse bien delante de la cámara. Además, tiene una argumentación bien organizada, pausada y fácil de seguir combinada con su discurso de buen tono, cautivo y muy bien vocalizado.

El libro y el documental transmiten la idea que el calentamiento global no es un tema político, sino que moral. Gore afirma que nadie tiene el derecho de comprometer la calidad de vida de las generaciones futuras a través de la escoja de un estilo de vida insostenible. Esto lleva otra vez el problema al alcance de el ciudadano común y correcto, que se ve obligado a reflexionar sobre su estilo de vida y evitar una conducta antiética.

Otro punto fuerte de las piezas de $\mathrm{Al}$ Gore desde el punto de vista de la comunicación es la comparación con atentados terroristas y la mención a la tragedia de 11 de septiembre de 2001. El presentador afirma que no tiene sentido gastar billones de dólares en la preparación contra ataques terroristas y no tener la misma preocupación con mitigar los impactos de un aumento considerable de temperatura. Con eso, Gore se mete en un tema tabú y extremamente impactante para los Estados Unidos y todo el mundo que está relacionado con el desmoronamiento de un símbolo y millares de muertos sobre el suelo americano, considerado intocable hace 10 años. Igualmente, al mencionar que en un futuro próximo el memorial del World Trade Center podrá ser inundado debido al aumento del nivel de mar, las piezas tocan profundamente los afectados por los atentados y despierta ganas de acción para evitar que la imagen del monumento desaparezca.

Gore también sabe hacer muy bien su interacción con el público y ordena su mensaje con excelencia, alternando datos científicos, opiniones personales, críticas, sugerencias y alertas. Él se muestra como una persona extremamente carismática sonriente y utiliza las bromas en el tiempo correcto para captar más la atención, impedir que los gráficos vuelvan a la presentación aburrida y ayudar a "break the ice". Todas estas habilidades hacen de Al Gore un atractivo orador y un personaje bastante querido, en general, por la audiencia de la película.

Igualmente, la manera con que la historia de Al Gore es desarrollada en el documental es bastante interesante. Una verdad incómoda es un documental pero utiliza muchos elementos del cine de ficción. Para ilustrar la presentación de Al Gore, el director optó por diferentes herramientas que varían entre imágenes alternándose rápidamente en momentos de tensión, pantallas negras con mensajes fuertes como "es la película de miedo más fuerte que verás", fotos antiguas y actuales de diversos espacios naturales exuberantes por el mundo exhibiendo las consecuencias de un planeta más cálido. Guggenheim desarrolla un enredo de ficción al largo de los 95 minutos de la película, siendo Al Gore el protagonista de una historia de una vida dedicada a la conservación ambiental. Y como complemento, la banda sonora se mezcla con excelencia a los imágenes mostrados, y alterna la transmisión de miedo, tensión y agobio con alegría y gracia. No se trata de una película de horror, como dice el autor, sino que de un drama personal con toques de acción, comedia, suspenso y romance. 
Sin embargo, la característica más importante presente en las obras de Al Gore está relacionada con el enredo de la historia. Tanto en el libro como en el documental, lo que se ve es la presentación sobre cambio climático mezclada con menciones constantes de su historia personal. En paralelo a la presentación corre las reflexiones de Gore sobre ética, el pasar del tiempo y momentos de su vida profesional y personal. Con eso, el público entra en contacto directo con la vida expuesta en las páginas y la pantalla y vive por un determinado tiempo todos los sentimientos de Gore, las aflicciones relacionadas al clima, la vida con su mujer Tipper, el accidente con el hijo más joven. Cuando menciona su vida por detrás de la política, Gore transforma Una verdad incómoda en una clásica historia de ficción en la cual él mismo ejerce el papel de héroe protagonista en una batalla de toda una vida contra los villanos escépticos con respecto a la crisis climática o cualquier uno que lleva un estilo de vida considerado insostenible para los estándares actuales. En las piezas, los espectadores prueban los cuestionamientos constantes de Gore consigo mismo y sus pensamientos sobre la relación entre la civilización y la Tierra y sus dudas sobre las acciones ciertas y erradas en la búsqueda de un futuro mejor para el planeta, ayudando así que el libro y el documental sean fáciles y agradables de ser apurados y Al Gore como un líder querido y apoyado.

\subsection{Críticas}

Durante mucho tiempo Al Gore señaló que las actividades humanas, sobretodo la quema de combustibles fósiles, están causando un calentamiento significativo en la Tierra, con potenciales resultados catastróficos. Sin embargo, mucho del material utilizado en la película no era nuevo. Como fue basado sobretodo en la presentación que Al Gore ya había dado muchas veces, los críticos de la película afirman que el político persistió en el error y no utilizó la oportunidad de la producción de el documental para corregirlo.

Las críticas recibidas por Al Gore están sobretodo relacionadas a un posible conflicto de intereses entre sus papeles de ambientalista y inversor en compañías de tecnologías verdes, la utilización de datos supuestamente erróneos, la gran cantidad de energía utilizada en sus negocios y su rechazo a debatir abiertamente con científicos y ambientalistas sobre el calentamiento global.

Según los críticos, entre ellos Bjorn Lomborg, el documental tiene muchos malentendidos, errores, medias verdades relacionadas a las causas y consecuencias del cambio climático. De acuerdo con H. Sterling Burnett (Burnett, 2006: web), Una verdad incómoda peca en diversos tópicos como el calentamiento de Tennessee, el derretir de la nieve en el monte Kilimanjaro, inundaciones causadas por el derretir de camadas de hielo polares y la unanimidad en la comunidad científica sobre el tema. En la película, Gore afirma que "el debate terminó", "la ciencia está definida" y "científicos están de acuerdo" la humanidad está causando el calentamiento global. La base científica más utilizada por Gore es un estudio publicado en la revista Science hecho por Naomi Oreskes, profesora en la Universidad de California en San Diego. Oreskes investigó los estudios relacionados al cambio climático de la base de datos del Instituto de Información Científica de los Estados Unidos entre los años de 1993 y 2003. 
Fueron analizadas 928 piezas en total, $25 \%$ de las cuales no mencionaron la influencia humana. Según Oreskes, $100 \%$ de los estudios que indicaron la influencia de la humanidad en el cambio climático aprueban explícitamente o implícitamente la visión de que los humanos son culpables del calentamiento actual. (Oreskes, 2004: web)

Otro tema que merece ser señalado está relacionado con las soluciones enseñadas por Al Gore. En el documental, él afirma que el calentamiento global es la amenaza más seria que la civilización humana ya enfrentó. Pero, la lista de acciones dada por Gore es blanda. El político no menciona medidas extremas y polémicas como tasas más grandes sobre la gasolina o cualquier combustible fósil o también como el racionamiento de gasolina, días obligatoriamente sin coches o aún la prohibición de carreras deportivas como la Formula 1. Igualmente, Gore sugiere que los Estados Unidos limite su emisiones de carbono según las reglas del protocolo de Kyoto, pero no menciona las dificultades relacionadas a esta acción, como bloqueos políticos, reducción de sueldos de electores o cualquier inconveniente para la sociedad.

Bjorn Lomborg es parte del grupo que afirma que Gore rechaza el debate. Lomborg ya le invitó para hablar sobre el tema en una conferencia en California. Gore, sin embargo, contestó diciendo que no participaría y utilizó la excusa de que "la comunidad científica ya debatió sobre este tema".

Por tratar como verdad absoluta datos sobre el clima terrestre que no son considerados como consenso en la comunidad científica y evitar el debate sobre los supuestos equívocos en sus piezas, Gore sufrió una gran pierda de credibilidad en los últimos años. El desgaste fue amplificado por la avalancha de críticas y nuevas incertidumbres sobre las mejores maneras de mitigar los impactos del cambio climático. Las críticas fueron lideradas por el grupo de los escépticos y por los que creen que el problema existe pero dudan de las soluciones propuestas y de los escenarios previstos para el planeta en los próximos años. Bjorn Lomborg es una de las personalidades más conocidas en este medio y sus ideas y piezas son el tema de análisis del próximo capitulo.

\section{Bjorn Lomborg y la batalla contra el apocalipsis}

"Si sólo escuchamos a los peores escenarios, estamos probablemente gastando todo nuestro dinero con las personas que gritan más alto"

\subsection{Biografía}

Bjorn Lomborg

Bjorn Lomborg es, desde 2005, profesor adjunto de la "Copenhagen Business School." Nació en enero de 1965, en Dinamarca. Es el organizador del "Copenhagen Consensus Center", centro que ya reunió algunos de los más renombrados economistas en el mundo (entre ellos 5 ganadores de premio Nobel) para definir las soluciones prioritarias para los mayores desafíos para la humanidad. Como reto, Lomborg pidió a los expertos para intentar contestar a la pregunta: "Con fuentes limitadas, como se puede hacer el bien más grande posible?" (Copenhagen Consensus, 2004: web)

Además de trabajar como director del "Copenhagen Consensus" y como profesor, es el autor de los best seller The skeptical environmentalist (2001) y Cool it (2007), en los cuales desafía las principales preocupaciones de la sociedad con respecto al 
medio ambiente y señala la necesidad de concentrar primero la atención en los problemas "más importantes".

Se graduó en Ciencias Políticas en 1991 y en 1994 adquirió su Ph.D. en el Departamento de Ciencias Políticas de la Universidad de Copenhagen. Entre 1994 y 1996, trabajó como profesor asistente en el Departamento de Ciencias Políticas de la Universidad de Aarhus hasta ser promovido, en 1997, a profesor asociado, puesto donde permaneció hasta 2005. Entre 2002 y 2004 asumió el puesto de Director General en el Instituto de Evaluación Medioambiental (Lomborg, 2006: web).

El proyecto del libro The skeptical environmentalist comenzó en 1998, cuando Lomborg trabajaba como profesor de estadística en el Departamento de Ciencias Políticas de la Universidad de Aarhus. Al publicar cuatro artículos sobre el estado del medio ambiente en un periódico del país, generó un fuerte debate que se extendió con la publicación de diversos otros artículos, los cuales promovieron la publicación de The skeptical environmentalist en 2001. (Lomborg, 2006: web)

Debido a la repercusión del primer libro, Lomborg se volvió un participante frecuente en los debates públicos. Sus comentarios aparecieron regularmente en diarios influyentes como The New York Times, Wall Street. Journal, USA Today, The Economist, Globe \& Mail, The Guardian, The Daily Telegraph, The Sunday Telegraph, The Times, The Australian, The LA Times y Boston Globe. Participó también en programas de grandes cadenas de televisión, como "Politically Incorrect", "60 minutes", "Larry King", "20/20" y "BBC Newsnight", además de otros en las cadenas CNN, BBC, CNBC, y PBS. (Lomborg, 2006: web)

Desde Febrero del 2002 a Julio del 2004, Lomborg fue director del Instituto de Evaluación Medioambiental de Dinamarca. Durante este período fue mencionado como una de las "50 Estrellas de Europa" por la revista Business Week. En Abril de 2004, fue clasificado como una de las 100 personas más influyentes del mundo por la revista Time.

En 2005, ganó el título de Líder Joven Global por el Forum Económico Mundial. En Junio de 2006, Lomborg repitió el proyecto "Copenhagen Consensus" con los principales representantes de las Naciones Unidas en lugar de economistas. Logró así reunir representantes de China, India y Estados Unidos, países que representan cerca de la mitad de la población mundial. El ranking de soluciones para los mayores desafíos del mundo fue el primero de esta categoría hecho por la ONU. Estas dos conferencias y sus resultados son los temas de los libros Global Crises, Global Solutions (2008) y How to Spend $\$ 50$ Billion to Make the World a Better Place (2006). El último proyecto "Copenhagen Consensus" ocurrió en mayo de 2008 y concluyó que el combate al calentamiento global tiene beneficios pero su prioridad, comparada con otros problemas, es baja y los proyectos como lo de optimización de la tasa de carbono (Copenhagen Consensus Center, 2009: web) y el Protocolo de Kyoto (Copenhagen Consensus Center, 2008: web) son las propuestas menos eficientes.

En 2008, fue mencionado como una de las "50 personas que podrían salvar el planeta" por el periódico británico The Guardian, uno de los "top 100 intelectuales públicos" por la revista Foreign Policy and Prospect y uno de las "75 personas más influyentes del siglo XXI" por la revista Esquire. 
En 2010 fue mencionado como uno de los "100 pensadores globales" por la revista Foreign Policy y en 2011 recibió el titulo de Pensador Líder en el Bloomberg Summit.

Después de unos años, los intereses de Lomborg se ampliaron desde simulaciones de estrategias y dilemas de acción colectiva hacia el uso de la estadística en el tópico del medio ambiente, que le motivaron a escribir The Skeptical Environmentalist, su libro más famoso en el área. Se trata de una obra cuestionada que afirma que las conclusiones existentes sobre los mayores problemas ambientales actuales no están correctas.

\subsection{La investigación del estado "real" del mundo}

El subtitulo del libro The skeptical environmentalist, "midiendo el real estado del mundo" se refiere a los informes State of the World, publicados anualmente desde 1984 por el instituto "World Watch". Lomborg considera los informes como "algunos de las mejores investigaciones académicamente más ambiciosas ya publicadas sobre política medioambiental" (Lomborg, 2001a: 258), pero, por otro lado, la crítica por la utilización de bases a corto plazo para prevenir consecuencias desastrosas en casos donde estudios de larga escala no tendrían las mismas conclusiones.

Con sus argumentos, Lomborg examinó en el libro una vasta lista de temas en las áreas relacionadas al medio ambiente, como economía ambiental y ciencia, y también presentó un largo abanico de conclusiones y recomendaciones. El trabajo de Lomborg desafió directamente ejemplos de preocupaciones verdes con su interpretación de datos de cerca de 3000 fuentes.

La idea del libro nació en una librería en Los Angeles en 1997, mientras el autor leía una entrevista del economista estadounidense Julian Simon, que afirmaba que mucho del conocimiento tradicional sobre el medio ambiente está basado simplemente en preconceptos y estadísticas pobres. Provocado, Lomborg, un antiguo miembro del Greenpeace y profesor de estadística empezó a dirigir grupos de estudios para analizar las afirmaciones de Simon, que descubrieron que el economista de hecho tenía razón en muchas de sus declaraciones.

Aunque afirme que la situación no está tan mal como se plantea, el autor señala que el mundo no está sin problemas, sino que sí mejorando en casi todas las áreas. Sin embargo, esto no quiere decir que todo está bien. Lomborg cree que saber el verdadero estado del mundo es importante porque define los problemas de la humanidad y muestra donde la acción es más necesaria. El autor justifica el título del libro porque se considera un ambientalista y se preocupa de la Tierra y el futuro de las próximas generaciones. Se clasifica también como escéptico por importarle "lo suficiente para no querer que la humanidad sólo actúe sobre los mitos de optimistas y pesimistas". (Lomborg, 2001a: 10)

El método analítico utilizado por Lomborg está basado en un análisis de coste-beneficio aplicado en economía, ciencias sociales y formulación y evaluación de políticas gubernamentales. Muchas de las observaciones de las letanías definidas por el autor tienen su base en datos de análisis estadístico y, por lo tanto, su trabajo debe ser considerado como de esta naturaleza. Como examina los costes y los beneficios de di- 
versos tópicos, puede ser considerado como un trabajo relacionado a la economía. Sin embargo, el libro es metodológicamente bastante variado entre diferentes disciplinas y combina la interpretación de datos con evaluaciones de los medios y el comportamiento humano, además de evaluaciones de teorías científicas para llegar a sus conclusiones.

Aunque sea bastante largo, con 515 páginas, con cada una dividida en 2 columnas de texto, y presente un gran número de fuentes, The skeptical environmentalist es de fácil lectura, lenguaje sencillo, y muy bien estructurado, pues casi siempre presenta los estudios evaluados en la generación de los argumentos.

Al llegar al trabajo final, Lomborg utilizó una metodología similar para cada uno de los tópicos y subtópicos principales de su trabajo. Avanza gradualmente desde algo general hacia algo específico, empezando con preocupaciones amplias como la contaminación y la energía, ramificándolas en subtópicos (contaminación del aire y combustibles fósiles) y luego identifica algunas preocupaciones compartidas mundialmente y sus respectivas fuentes. A partir de ahí, Lomborg elige los datos disponibles que considera más fiables y razonables y después los evalúa para intentar probar su posición. En cada caso, sus cálculos encuentran que las alegaciones no tienen base y que se tratarían de exageraciones o retratos negativos de situaciones que no estarían empeorando, sino que mejorando, en realidad.

Lomborg establece el llamado "verdadero estado del mundo" y examina una variedad de teorías, tecnologías, implementación de estrategias y costes y sugiere caminos alternativos para las situaciones que no estarían tan mal en cada uno de los tópicos y subtópicos del libro.

“The Litany" (Lomborg, 2001a: 327) o la letanía citada por Lomborg se relaciona con áreas bastante diversas. El autor cita fuentes mundialmente aceptadas como el Gobierno de los Estados Unidos, las agencias de las Naciones Unidas y da preferencia a datos de escala global a largo plazo. Los principales temas evaluados son la prosperidad humana desde un punto de vista económico y demográfico, la prosperidad humana desde un punto de vista ecológico, la contaminación como una amenaza a la prosperidad humana y futuras amenazas para la prosperidad humana. Su argumento principal es que la mayoría de los problemas medioambientales como la contaminación, escasez de agua, deforestación y pérdida de biodiversidad, así como el aumento de población, hambre y SIDA son áreas específicas y relacionadas con la pobreza. Por lo tanto, los desafíos para la prosperidad humana son temas esencialmente logísticos y pueden ser solucionados ampliamente a través del desarrollo social y económico. Con respecto a problemas de nivel global, como la utilización de combustibles fósiles y el cambio climático, Lomborg afirma que estos temas son normalmente exageraciones y que las políticas recomendadas para solucionarlos son casi siempre inapropiadas en comparación con las alternativas posibles.

Cuando discute sobre las exageraciones con respecto a las amenazas para la prosperidad humana, Lomborg propone algunas recomendaciones y evaluaciones de análisis de beneficios y gastos. El autor cita diversos ejemplos, como la ligación del desarrollo del cáncer con la utilización de pesticidas, la escasez de agua o la perdida de biodiversidad. Pero mientras admite los problemas, el afirma también que no se tra- 
tan de las catástrofes definidas por muchos autores (como Al Gore por ejemplo) y que tienen pocos impactos para la prosperidad humana. (Lomborg, 2001a: 72)

Sin embargo, el tema principal examinado por Lomborg es el calentamiento global. Desde el principio, el autor afirma aceptar "la realidad del calentamiento global antropogénico", pero se refiere a un número de incertidumbres en las simulaciones digitales sobre el cambio climático y algunos aspectos de la utilización de los datos. El autor no se concentra principalmente en la ciencia del calentamiento global, sino que en la política y las acciones políticas relacionadas a los descubrimientos científicos. Lomborg afirma que al llevar en consideración la cantidad de reducción de gases de efecto invernadero necesaria, el Protocolo de Kyoto es considerablemente insuficiente. Lomborg sugiere que la importancia del calentamiento global en términos de prioridades políticas debe ser menor comparada con otros problemas como pobreza y la proliferación de enfermedades, que tienen impactos más inmediatos en términos de bienestar humano y para el medio ambiente. El autor, por lo tanto, propone un análisis global de beneficios y gastos antes de se tomar decisiones.

\subsection{EI mensaje tranquilizante de Cool It}

En septiembre de 2007, Lomborg publicó el libro Cool it, que proporcionó una nueva perspectiva para el debate sobre el calentamiento global, basada en las necesidades humanas y cuestiones ambientales. En la pieza, Lomborg afirma que muchas de las caras acciones mundiales que están siendo consideradas actualmente para parar el calentamiento global tendrán un coste de miles de millones de euros no están basadas en estudios estrictamente científicos, sino que en suposiciones emocionales y no van a tener mucho impacto en la temperatura global por centenas de años. Para el danés, mejor que empezar con procedimientos radicales, seria utilizar los fondos para problemas más inmediatos como la lucha contra la malaria y el SIDA así como asegurar y mantener una fuente de agua segura y limpia (Lomborg, 2007a: 38). Lomborg afirma que estos problemas podrían ser solucionados con apenas una fracción del coste y salvar millones de vidas en una generación. Sin embargo, el autor no limita su discusión al cambio climático y presenta una segunda generación de pensamientos sobre el calentamiento global, que cree que el pánico no es garantía de solución ni tampoco constructivo para lidiar con los problemas de la humanidad.

El documental Cool it se estrenó en 2010 y está basado en el libro homónimo y en presentaciones hechas por Bjorn Lomborg. Ondi Timoner, cineasta premiada, viajó por el mundo con Lomborg para explorar los hechos reales con relación al calentamiento global y a sus impactos.

El documental es mas reciente que Una verdad incómoda y no ha tenido, hasta el momento, el mismo impacto mundial. Lomborg sigue siendo conocido por el libro The skeptical environmentalist. Sin embargo, Cool it es una buena adaptación cinematográfica de los ideales del danés y fue minuciosamente elaborado pensando en la fácil comprensión del mensaje.

La directora Ondi Timoner tenía la intención de presentar Lomborg y sus problemas al público. Su estrategia fue empezar a hacer un documental rápido, efectivo, con movimiento, música y gráficos de fácil comprensión. La opción por un tour de varias 
soluciones practicas que se están investigando y desarrollando actualmente es bastante interesante, dinámica e inspira la acción del público. Cuando empieza a mencionar sobre las nuevas tecnologías que pueden ayudar a mitigar el cambio climático, surge una canción animada y dinámica de fondo, que ayuda a transmitir el mensaje que el mundo no está caminando hacia el colapso y a tranquilizar el público.

En esta presentación de Lomborg, el espectador ve un personaje simpático, inteligente, valiente y de espíritu juvenil. El protagonista es visto varias veces utilizando bicicletas o transporte público e intenta mostrarse personalmente comprometido con la causa que defiende. La directora exhibe la historia de un intelectual mal comprendido y cruelmente atacado por un vasto número de científicos que parecen tener miedo de encarar los supuestos hechos revelados por Lomborg. Las declaraciones del autor sobre este tema parecen ser bastante sinceras y enseñan una persona triste con las duras críticas pero con muchas ganas de ayudar el mundo y hacer las cosas de la mejor manera posible.

Así como en Una verdad incómoda, el personaje principal tiene considerable importancia para el éxito de la transmisión del mensaje. Lomborg tiene apariencia jovial, está siempre usando ropas casuales (zapatillas, vaqueros, camisa neutra y una mochila en la espalda) en vez de trajes y sus respuestas son siempre rápidas y directas al punto. Su discurso también es extremamente bien organizado, vocalizado, argumentado, pausado en las horas ciertas y muy fácil de ser seguido.

Cuando visita una chabola en Nairobi donde las personas viven sin necesidades básicas como trabajo, electricidad, comida y vivienda decientes, Lomborg intenta escuchar lo que niños y adultos piensan sobre el problema. El danés es visto jugando con niños, ayudando a buscar agua, distribuyendo comida o simplemente intentando escuchar lo que piensan y desean. Todas estas imágenes ayudan a desmitificar la idea de un monstruo que gran parte del público general tenía sobre Lomborg.

Sin embargo, lo que Lomborg parece ser capaz de hacer mejor que Al Gore es reconocer sus errores y estar abierto a debates. Mientras el estadounidense se recusa a discutir públicamente sus piezas, Lomborg parece estar siempre abierto a críticas, hecho que se refleja en su presencia constante en los medios, la escoja de declaraciones de expertos contra sus ideales para su película y la creación de las sesiones de errores y críticas en su pagina web oficial (Lomborg.com, 2003: web).

Al largo de sus 88 minutos, la película mezcla declaraciones de Lomborg y de expertos con presentaciones y participaciones de Lomborg en los medios y desarrolla así una historia interesante de un profesor ambientalista en su batalla contra las falsas realidades. El tiempo del documental es ideal. Casi una hora y media es suficiente para transmitir un mensaje y no aburrir a los espectadores con mucha información.

Cool it merece destaque también porque, así como The Skeptical environmentalist, transmite esperanzas y fornece un tiempo de respiración y reflexión para los espectadores ahogados con las amenazas de Apocalipsis encontradas en los medios constantemente. Lomborg rebate las afirmaciones apocalípticas de Al Gore y afirma que asustar al público no es la mejor manera de comunicar el problema. Cuando visita una escuela primaria en el Reino Unido el autor se depara con niños aterrorizados por las posibles consecuencias del aumento de la temperatura global (muchas expuestas por 
Al Gore) y hace una interesante comparación con los niños de Nairobi que están mucho más preocupados con el sistema sanitario, enfermedades y vivienda.

El título Cool it es sencillo y llama la atención del público. Como se puede percibir fácilmente, se trata de un juego de palabras que mezcla la expresión que en inglés es utilizada informalmente para decir a alguien que se tranquilice, con la necesidad de enfriar el planeta. El símbolo utilizado con una bombilla fluorescente, también es bastante creativo y tiene un fuerte poder de comunicación pues hace un juego con el símbolo de la eficiencia energética utilizado representar nuevas ideas, imagen que comúnmente es asociada con una bombilla común de filamento.

Otra característica del documental que contribuyó a su éxito fue la elección de declaraciones de expertos extremamente interesantes, importantes y variados para dar base a la argumentación de la pieza. Algunos de los nombres vistos en Cool it son Freeman Dyson, teórico, físico y matemático conocido por sus trabajos en ingeniería nuclear y ex profesor del Instituto de Estúdios Avanzados de Princeton; Jagdish Bhagwati, profesor de economía en la Universidad de Columbia; Daniel Nocera, profesor de energía y química en el Instituto de Tecnología de Massachussets; Rajendra Pachauri, director del IPCC; Gywn Prins, director del programa Mackinder de London School of Economics para el estudio de eventos de largas olas; Stephen Salter, profesor de Ingeniería y Design de la Universidad de Edimburgo e inventor del Salter Duck, un dispositivo que convierte la energía de las olas en electricidad; Stephen Schneider, profesor de biología medioambiental y cambio global en la Universidad de Stanford y coordinador del IPCC; Vernon Smith, profesor de economía en la Universidad de Chapman y ganador del Nobel Memorial en ciencias económicas en 2002; David Vaughan, un científico climático del British Antartic Survey (Cool It The Movie, 2010).

Sin embargo, así como en Una verdad incómoda, lo que vuelve el documental extremamente interesante es el toque personal del protagonista. Cool it no es apenas un documental lleno de declaraciones, gráficos y datos. Lo que llama la atención de los espectadores es la historia personal de Lomborg, un intelectual común e inteligente, que cuida de la madre mayor y vive preocupado no sólo por el medio ambiente, pero con los problemas del mundo en general. Él se muestra preocupado por frenar el cambio climático pero también mejorar la calidad de vida de la sociedad y disminuir índices de hambre, pobrezas y la propagación de enfermedades. Los cuestionamientos constantes sobre la relación entre civilización y la Tierra presentes en las piezas de $\mathrm{Al}$ Gore dan sitio a los pensamientos de Lomborg sobre como ayudar mejor al mundo y comprobar que sus ideales son correctos y que las críticas recibidas no tienen fundamentos. Cuando visita las escuelas primarias en Kenia y Reino Unido, se muestra cómodo al hablar con niños, consiente de los problemas que tienen que enfrentar y emocionado por ver tantos niños con hambre, actitudes que hacen el espectador conmoverse con los hechos y tener ganas de actuar para ayudar a reverter la situación. Todos estas características ayudan en la composición del personaje héroe Bjorn Lomborg como un líder intelectual querido por el público general. 


\subsection{Críticas}

Tras la publicación de The Skeptical Environmentalist, Lomborg fue acusado de deshonestidad científica. Diversos cientistas ambientales denunciaron el libro en el Comité Danés de Deshonestidad Científica (DCSD), una agencia ligada al Ministerio de Ciencia, Tecnología e Innovación (MSTI) de Dinamarca. Las acusaciones decían que el libro tenía datos deliberadamente mal interpretados y conclusiones equivocadas.

En 2003, el DCSD decidió que el libro es científicamente deshonesto pero consideró Lomborg no culpable debido al nivel de experticia de los campos en cuestión. El libro fue citado por fabricación de datos, descarte selectivo de resultados no deseados, uso deliberadamente equivocado de métodos estadísticos, interpretación destorcida de las conclusiones, y plagio.

En el mismo año, Lomborg empezó con un proceso de queja junto al MSTI contra la decisión del DCSD. Luego, el Ministerio anuló la decisión tomada por el comité por no proporcionar argumentos suficientes sobre las acusaciones de errores, de no utilizar un estándar para definir la "buena practica científica" y por una falta de claridad en la definición de deshonestidad científica. En 2004, el DCSD decidió no actuar formalmente con respecto a las nuevas quejas sobre The skeptical environmentalist.

Por otro lado, la comunidad científica criticó el libro también desde los ángulos de los datos, del análisis y de las conclusiones. En 2002, la revista Scientific American publicó "La matemática engañosa sobre la Tierra", una serie de papers escritos por diversos científicos que afirmaron en común que The skeptical environmentalist no representa ni las evidencias científicas ni la opinión de la comunidad científica (Scientific American, 2002: web). Tras negar pedidos de defensa de Lomborg, la revista publicó cuatro meses después un pequeño articulo donde el danés señaló su opinión punto por punto y luego rebatió su descontentamiento por la desproporción de longitud textual de su defensa en comparación con las más de 30 paginas que recibió de criticas (Lomborg, 2002:web).

La revista Nature también publicó un resumen critico del libro de Lomborg, donde Stuart Pimm, miembro del Centro de Investigación Ambiental y Conservación de la Universidad de Columbia y Jeff Harvey, y representante del Instituto de Ecología de Holanda escribieron: "el texto utiliza la estrategia de aquellos que, por ejemplo, afirman que hombres gays no están muriendo de SIDA o que judíos no fueron discriminados por Nazis para la exterminación" (Kellow, 2007a: 103).

Quizás la critica más directa y expuesta fue la creación, en 2001, de la pagina web anti-lomborg.com. El dominio digital fue desarrollado por diversos académicos, escritores y activistas ambientales de Oxford, todos insatisfechos con las supuestas imprecisiones de la serie de artículos "green wash" publicados en el periódico The Guardian en 2011 y firmados por el danés. El objetivo de la pagina web es "discutir teorías alternativas sobre el medio ambiente, calentamiento global, desperdicio, administración y fuentes alternativas de energía, como el bio diesel o la energía solar y todos los otros tópicos relacionados. El objetivo no es atacar Bjorn Lomborg, sino que si señalar y discutir algunos equívocos de sus análisis" (Anti-lomborg, 2001: web). "Este libro probablemente atrajo más la atención del publico que cualquier otro libro sobre los problemas ambientales del mundo desde Limits to Growth." (Pannel, 2008: web). 
La conclusión de Lomborg es que, por ahora, en vez de pagar con costes altísimos, la sociedad debe aumentar fuertemente las inversiones en I+D+i para acelerar el desarrollo de fuentes de energía mejores y económicamente competitivas. Por una fracción del coste de Kyoto, tal innovación podría producir tecnologías que pueden ser ampliamente adoptadas con bajos costes.

Las conclusiones de Lomborg muchas veces parecen ser bastante genéricas. La perspectiva global de los problemas oculta las cuestiones ambientales regionales que pueden también ser importantes. Es verdad que el libro critica algunas de las decisiones políticas tomadas en las últimas décadas con respecto al medio ambiente. Sin embargo, las conclusiones del libro no deben ser sólo criticadas o utilizadas como una Biblia sobre el calentamiento global, sino que si como refuerzo para la importancia de buscar los mejores datos científicos disponibles, en vez de aceptar la inercia convencional de algunas falsas realidades.

\section{Consideraciones finales}

El presente artículo ha tenido desde el principio la intención de contribuir con la calidad de las publicaciones sobre los temas relacionados al medio ambiente destinadas al público general. Este objetivo fue considerado relevante debido a la complejidad de las ciencias del clima y a la gran diferencia de conocimiento entre los expertos y el público general. Clarificar y facilitar este proceso de comunicación es crucial, pues simplifica las tomas de decisión políticas e inspira primordiales acciones de cambio en la sociedad.

Tras la conclusión de los análisis propuestos, es posible afirmar que las ciencias del clima y la historia del planeta son extremamente complejas y difíciles de se comunicar al público general. Este tema envuelve diversas áreas distintas de la ciencia y conocimientos bastante profundizados. A nivel de hoy, no existe un consenso sobre los posibles impactos concretos de un cambio en la temperatura global y, por lo tanto, considerar un solo estudio, por más bien evaluado que sea, como una verdad absoluta constituye a un error. Con respecto a la problemática inicial, que buscaba la mejor manera de transmitir el mensaje ambiental en los medios, se puede afirmar que no es posible establecer un guía concreto que pueda garantizar el éxito total de una pieza de comunicación. No obstante, es posible señalar algunas herramientas claves para comunicar de la mejor manera este tema específico y que fueron destaques en los análisis hechos en el presente trabajo. El estudio del receptor (en este caso el público general) es siempre crucial para que se pueda adaptar el mensaje de la mejor manera posible. Cómo se trata de un tema complejo, es importante también utilizar y citar las mejores y más diversas fuentes posibles, para que la audiencia atribuya relevancia al contenido del mensaje. Igualmente, el emisor debe ser cercano, claro, simpático, tener una buena imagen y el discurso debe ser sencillo y articulado. Se deben evitar exageraciones, alarmar demasiadamente al receptor y, claro, mentiras.

Sin embargo, la característica más importante encontrada fue el toque personal dado por los personajes. Todas las piezas analizadas son atractivas para transmitir el problema mezclado con historias de vida de personas interesantes e inteligentes. Esto hace que mismo alguien que no tenga ningún interés sobre el tema esté atraído por las 
historias interesantes que estas piezas proporcionan y reciba el mensaje inicialmente propuesto.

\section{Referencias bibliográficas}

ANTI-LOMBORG. (2001): “Anti-lomborg": http://www.anti-lomborg.com/ [fecha de consulta: 16 de octubre de 2011]

BURNETT; H. Sterling (2006): “The truth about an inconvenient truth", en National Center for Policy: http://www.ncpa.org/pub/ba561 [fecha de consulta: 25 de septiembre de 2011]

COPENHAGEN CONSENSUS CENTER (2004): “Copenhagen Consensus 2004", en Copenhagen Consensus: http://copenhagenconsensus.com/Projects/Copenhagen\%20Consensus\%202004-1.aspx [fecha de consulta 11 de octubre de 2011]

COPENHAGEN CONSENSUS CENTER (2008): "Findings of the Expert Panel”, en Fix the Climate: http://fixtheclimate.com/uploads/tx_templavoila/CC_FINAL_RESULT_02.pdf [fecha de consulta: 17 de octubre de 2011]

COPENHAGEN CONSENSUS CENTER (2009): “Advice for politicians”, en Fix the Climate: http://fixtheclimate.com/ fecha de consulta [10 de octubre de 2011]

GORE, Al (2006a): An inconvenient truth. Rodale

KELLOW, Aynsley (2007): Science and public policy. Northampton, Edward Elgar Publishing Limited

LOMBORG, Bjorn (2001a): The skeptical environmentalist. Cambridge University Press

LOMBORG, Bjorn (2003): "Errors and corrections", en Lomborg: http://lomborg.com /publications/the_skeptical_environmentalist/errors/[fecha de consulta: 14 de octubre de 2011]

LOMBORG, Bjorn (2007a): Cool it. Random House

LOMBORG, Bjorn (2008): "Biography”, en Lomborg: http://lomborg.com/about/biography/ [fecha de consulta: 14 de octubre de 2011]

LOMBORG, Bjorn (2010): “Featured scientists”, en Cool It - The Movie: http://coolitthemovie.com/about\#featuredscientists [fecha de consulta: 16 de octubre de 2011]

NOBEL PRIZE FOUNDATION (2007): “Al Gore - Biography”, en Nobel Prize: http://www.nobelprize.org/nobel_prizes/peace/laureates/2007/gore-bio.html [fecha de consulta: 10 de septiembre de 2011]

ORESKES, Naomi (2004): "Beyond the Ivory Tower: The Scientific Consensus on Climate Change". Science Magazine, Vol. 306, n¹. 5702, pp. 1686.

PANNELL, David J. (2008): "Book review”, en Dpaneel: http://dpannell.fnas .uwa.edu.au/lomborg.htm [fecha de consulta: 15 de octubre de 2011]

SCIENTIFIC AMERICAN MAGAZINE (2002): "The misleading math about the Earth”. Scientific American Magazine, January 2002 issue 
SCIENTIFIC AMERICAN MAGAZINE (2002): “The skeptical environmentalist replies”. Scientific American Magazine, May 2002 issue

TAKE PART (2007): “Al Gore”, en Climate Crisis: http://www.climatecrisis.net /an_inconvenient_truth/about the_film.php [fecha de consulta: 13 de septiembre de 2011$]$

TAKE PART (2007): “Impacts", en Climate Crisis http://www.climatecrisis.net/an_inconvenient_truth/the_impact.php [fecha de consulta: 20 de septiembre de 2011] 